\title{
Seizure Prediction in Epilepsy and Reaction Diffusion Cellular Nonlinear Networks (RD-CNNs)
}

\author{
A. Osman, V. Senger, R. Tetzlaff \\ Faculty of Electrical Engineering and Computer Engineering, \\ Institute of Circuits and Systems, \\ Technische Universität Dresden, 01062 Dresden, Germany \\ Alsheima.Osman_Gesmalla@mailbox.tu-dresden.de
}

\begin{abstract}
:
The seizure prediction problem has been addressed by many researchers from very different fields for more than three decades. The vision of an implantable seizure prediction device may become reality now: the first clinical study of such a device has been realized very recently and other realizations are not far behind. Despite promising first results, however, up to now there is no method allowing an automated seizure prediction with sufficient sensitivity and specificity required for broad clinical application..
\end{abstract}

Key words: EEG, Epilepsy, Seizure, Prediction, and RD-CNNs.

\section{Introduction}

Our group has more than 20 years of expertise in the development of seizure prediction methods drawn from many fields of signal processing and nonlinear dynamics. In our investigations, we focus on algorithms based on CNN. The paradigm of CNN was first introduced in 1988 [1] and later extended to an inherently parallel processing framework called the CNN-UM by Chua and Roska [2]. These programmable devices are implemented on low-power consuming platforms having the necessary features for the realization of implantable devices. Algorithms for seizure prediction based on CNN have been discussed in a large number of publications from various authors [5 - 10].

In our group, an estimation of the correlation dimension of EEG signals, measures based on the approximation of statistical signal properties by analyzing level-crossings of the signal as black-and-white-pixel representations have been introduced in the past. Despite promising first results based on short-time recordings of EEG, a detailed statistical analysis of those measures revealed that they are not suitable to the prediction of seizures if taken into consideration without additional information. Additionally, methods based on linear and nonlinear CNN signal prediction have been suggested. Here, the prediction error of a multivariate signal prediction is taken into account as a seizure precursor, combined with a pre-processing by Principal Component Analysis (PCA) and a post-processing by a level-crossing analysis of the prediction error [3].

\section{Seizure Prediction and RD-CNNs}

A very promising measure is based on the modelling of brain electrical activity based on Reaction-Diffusion Cellular Nonlinear Networks (RD-CNNs). Reaction-Diffusion systems can be used to describe a variety of nonlinear phenomena, such as pattern formation and chaotic behavior. An example model of a reaction-diffusion system in biology is the FitzHugh-Nagumo nerve conduction model, in chemistry, the Oregonator model for the Belousov-Zhabotinsky reaction follows similar laws, and in physics, plasma systems can be described by Reaction-Diffusion equations.

RD-CNNs are RD-CNNs are space-discretized models of partial differential equations describing the reaction-diffusion physical processes. For these the Local Activity Dogma can be used to analyse emergent complex behaviour. In order to derive a CNN based model with desired properties, a concrete representation of the reaction function has to be chosen. For this, polynomial weight functions are chosen which are capable of representing a wide range of nonlinear functions by power series expansion. Based on the assumption that the dynamics behind intracranial EEG recordings can be described by reactiondiffusion-networks an algorithm for the blind 
identification of RD-CNN has been studied. A numerical model of a multi-layers RD-CNN has been developed and polynomial weight functions have been implemented to ensure the applicability of the model to a wide range of problems [5]. To test the model, the FitzhughNagumo-equations have been used as a benchmark process. The model has been able to reproduce time series resulting from these equations with great accuracy, both using an analytically derived RD-CNN model as well as a model created within a supervised optimization process [8].

Additionally, a modelling technique allowing the identification of model parameters in Laplace domain as shown in Fig. 1 has been developed and thoroughly tested. Results so far indicate that a modelling of brain electrical activity using our newly developed method is not only accurate but also less computationally demanding than the models considered so far [4]. Therefore, we are about to underline an algorithm for a reliable prediction of epileptic seizures.

\section{References}

[1] L. Chua and L. Yang, Cellular Neural Networks Theory, IEEE Trans. on Circuits and Systems, 35(10):1257-1272, 1988.

[2] L. Chua, T. Roska, Cellular Neural Networks and Visual Computing, Foundations and Applications 2005.

[3] Vanessa Senger, Ronald Tetzlaff, Cellular Nonlinear Network - based signal prediction in epilepsy: Method comparison, Proc. of the International Symposium of the Circuits and Systems Society of the IEEE ISCAS 2015, Lisbon, 2015.

[4] Alsheima Osman, Ronald Tetzlaff, Modelling Brain Electrical Activity by Reaction Diffusion Cellular Nonlinear Networks (RD-CNN) in Laplace Domain", CNNA 2014, USA.

[5] Gollas, F., Niederhöfer, C., Tetzlaff, R., Toward an autonomous platform for spatio-temporal EEG-signal analysis based on Cellular Nonlinear Networks, International Journal of Circuit Theory and Applications, Special Issue: Cellular Wave Computing Architecture, Volume 36, Issue 5-6, pp. 623-639, June 2008.

[6] Gollas, F., Tetzlaff, R.: Analysis of Local Activity in Reaction-Diffusion Networks Identifying EEG Signals in Epilepsy, 19th European Conference on Circuit Theory and Design (ECCTD2009), Antalya, Turkey, August 2009.

[7] Fischer, P., Gollas, F., Kunz, R., Niederhöfer, C., Reichau, H., Tetzlaff, R., Cellular Neural Networks and Seizure Prediction: An Overview, in: Schelter, B., Timmer, J. , Schulze-Bonhage, A. (ed.), Seizure Prediction in Epilepsy, From Basic Mechanisms to Clinical Applications, Wiley- $\mathrm{VCH}$,
Berlin, 2008, pp. 117-129, ISBN: 978-3-52740756-9.

[8] Gollas, F., Tetzlaff, R.: Modelling Complex Systems by Reaction Diffusion Cellular Nonlinear Networks; Proceedings of the IEEE International Workshop on Cellular Neural Networks and their Applications (CNNA 2005), Hsinchu, Taiwan, pp. 227-231, 2005.

[9] C. Niederhöfer, F. Gollas, R. Tetzlaff, Dynamics of EEG-signals in epilepsy: spatio temporal analysis by Cellular Nonlinear Networks, 1-42441342-7/07/\$25.00 C2007 IEEE.

[10] C. Niederhöfer, F. Gollas, R. Tetzlaff, EEG analysis by multi-layer Cellular Nonlinear Networks (CNN), 1-4244-0437-1/06/\$20.00 C)2006 IEEE.

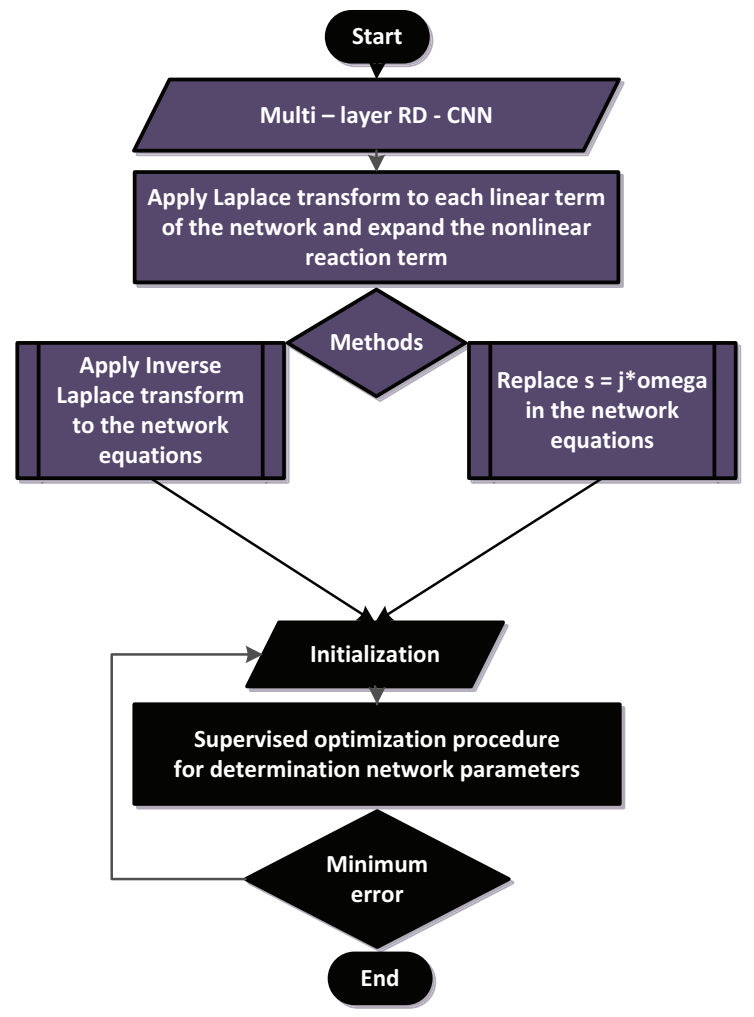

Fig. 1. A modelling technique using RD - CNNs allowing the identification of model parameters in Laplace domain. 